\title{
Effect of electron-phonon interaction on the shift and attenuation of optical phonons
}

\author{
L. A. Falkovsky \\ L. D. Landau Institute for Theoretical Physics, 2 Kosygin St., Moscow 117334, Russia
}

\begin{abstract}
Using the Boltzmann equation for electrons in metals, we show that the optical phonons soften and have a dispersion due to screening in agreement with the results reported recently [M. Reizer, Phys. Rev. B 61, 40 (2000)]. Additional phonon damping and frequency shift arise when the electron-phonon interaction is properly included.

PACS numbers: 63.20.Dj, 63.20.Kr, 78.30.-j
\end{abstract}

Despite attracting considerable interest for half a century since the pioneering work by Fröhlich, the problem of electron-phonon interaction is still far from being solved. Migdal 11 developed a consistent many-body approach based on the Fröhlich Hamiltonian for interaction of electrons with acoustic (sound) phonons. As Migdal showed ("the Migdal theorem"), the vertex corrections for acoustic phonons are small by the adiabatic parameter $\sqrt{m / M}$, where $m$ and $M$ are the electron and ion masses, respectively. The theory described correctly the electronic lifetime, renormalization of the Fermi velocity $v_{F}$ and acoustic phonon attenuation but resulted in a strong renormalization of the sound velocity $\tilde{s}=s(1-2 \lambda)^{1 / 2}$, where $\lambda$ is the dimensionless coupling constant. For sufficiently strong electron-phonon coupling $\lambda \rightarrow 1 / 2$, the phonon frequency approached to zero marking an instability point of the system. Instead, one would intuitively expect the phonon renormalization to be weak along with the adiabatic parameter.

This discrepancy was resolved by Brovman and Kagan [2] almost a decade later (see also [3]). They demonstrated the shortcomings of the Fröhlich model that gave an anomalously large phonon renormalization. Employing the Born-Oppenheimer (adiabatic) approximation (see, e.g., (4]), they found that there are two terms in the second order perturbation theory, which compensate each other making a result small by the adiabatic parameter. Namely, when calculating the phonon self-energy function $\Pi(\omega, k)$ with help of the diagram technique, one should eliminate an adiabatic contribution of the Fröhlich model by subtracting $\Pi(\omega, k)-\Pi(0, k)$.

The interaction of electrons with optical phonons was first considered by Engelsberg and Schrieffer [5] within Migdal's many-body approach for dispersionless phonons. They predicted a splitting of the optical phonon at finite wavenumbers $k$ into two branches. Ipatova and Subashiev [6] calculated later on the optical phonon attenuation in the collisionless limit and pointed out that the Brovman-Kagan renormalization should be carried out for optical phonons in order to obtain correct phonon renormalization. In the paper [7], Alexandrov and Schrieffer corrected the calculational error of Ref. [5] and argued that no splitting was found in fact. Instead, they predicted an extremely strong dispersion of optical phonons, $\omega_{k}=\omega_{0}+\lambda v_{F}^{2} k^{2} / 3 \omega_{0}$, due to the coupling to electrons. No such a dispersion has ever been observed experimentally. The usual dispersion of optical phonons in metals has the order of the sound velocity. In a recent paper, Reizer [8] stressed the importance of screening effects which should be taken into account. The works [7, [8] are limited to the case of collisionless both electron and phonon systems. Moreover, only the phonon renormalization was considered with no results available for the attenuation of optical phonons.

A different from many-body technique semiclassical approach based on the Boltzmann equation and the equations of the elasticity theory was developed in the papers by Akhiezer, Silin, Gurevich, Kontorovich, and many others (we refer the reader to the review [9]). This approach was compared with various experiments, such as attenuation of sound waves, effects of strong magnetic fields, crystal anisotropy, and sample surfaces on the sound attenuation, and so on. It can be applied to the problem of the electron-optical-phonon interaction [10] as well. In the present paper we develop a theory for both the attenuation and frequency shift of optical phonons with account for effects of the Coulomb screening as well as collisions in the electron and phonon systems.

It is instructive to recapitulate the results of the dynamical theory of elasticity for the renormalization of the sound velocity $\delta s=\tilde{s}-s$ and acoustic attenuation $\Gamma$ in metals. For a phonon with a wavenumber $k$ and frequency $\omega_{k}=s k$, they are given by [9]

$$
\frac{\delta s}{s}-i \frac{\Gamma}{\omega_{k}}=\lambda \begin{cases}\frac{s^{2}}{v_{F}^{2}}-i \frac{\pi s}{2 v_{F}} & \text { for } k v_{F}>\left|\omega_{k}+i \gamma\right|, \\ \frac{\omega_{k}+i \gamma}{\omega} & \text { for } k v_{F}<\left|\omega_{k}+i \gamma\right|,\end{cases}
$$

where $\gamma$ is the electronic scattering rate, and the dimensionless coupling constant $\lambda$ is proportional to the electronic density of states $\nu_{0}$ at the Fermi surface (for the isotropic case $\nu_{0}=m^{*} p_{F} / \pi^{2}, m^{*}$ is the effective electron mass) and to the squared deformation potential $\zeta_{i k}$. The deformation potential describes the change in the spectrum of electrons subject to lattice deformation $\varepsilon(\mathbf{p}, \mathbf{r}, t)=\varepsilon_{0}(\mathbf{p})+\zeta_{i k}(\mathbf{p}) u_{i k}(\mathbf{r}, t)$, where $u_{i k}$ is the strain tensor. Equations (11) give the correct answers in various known regimes: for the sound attenuation in the hydrodynamic limit $\left(\omega_{k} \ll \gamma\right.$ and $k \rightarrow 0$ ), for the zero-sound 
$\left(\omega_{k} \gg \gamma\right.$ and $k \rightarrow 0$ ), for the Landau damping in the ballistic limit $\left(k v_{F} \gg\left|\omega_{k}+i \gamma\right|\right)$. In the latter case, both the sound velocity shift and attenuation are small by the adiabatic parameter $s / v_{F}$ contrary to the results of the Fröhlich model. Note also that Eqs. (1) show hardening of the phonon frequency due to the electron-phonon interaction in contradiction with Migdal's result.

For the case of optical phonons, two known types of the electron-phonon interaction are the deformation potential and the interaction with the electrical polarization induced by optical vibrations. We consider here for simplicity a cubic crystal with two different atoms in a unit cell. Then, there are three optical modes, and the interaction with the induced polarization has the Fröhlich form:

$$
\varepsilon(\mathbf{p}, \mathbf{r}, t)=\varepsilon_{0}(\mathbf{p})+\zeta(\mathbf{p}) \nabla \cdot \mathbf{u}(\mathbf{r}, t)
$$

where the scalar function $\zeta(\mathbf{p})$ of the electron momentum is the coupling with the optical displacements $\mathbf{u}$. In order to compare our results with previous ones, here we consider the interaction in the same form (2) as in Refs. [7], [8]. One can see that principal characteristic features of the phenomenon are retained for the deformation interaction, $\delta \varepsilon=\zeta_{i}(\mathbf{p}) u_{i}(\mathbf{r}, t)$, where the coupling is a vector function. The distinction is that only the longitudinal mode interacts with electrons in the case of induced polarization (2), and the interaction approaches zero in the long-wave limit. Therefore, we concentrate on the propagation of the longitudinal mode along the symmetry axis when this mode is not mixed with transverse ones. Note also that the electric field plays an important role especially when the different atoms are in the unit cell so that the dipole moment is excited under the atom vibrations. At last, the optical phonons have always the so-called natural width $\Gamma^{\text {nat }} \sim \omega_{0} \sqrt{m / M}$. The natural width results from decay processes into two (or more) acoustic or optical phonons, which are possible even at zero temperature.

The main point of the theory is the equation of motion in the long-wave approximation $(k \ll 1 / a)$ for the Fourier components of the optical-phonon displacement $u_{j}$ :

$$
\begin{array}{r}
\left(\omega_{k}^{2}-i \omega \Gamma^{\mathrm{nat}}-\omega^{2}\right) u_{j}(\mathbf{k}, \omega)=\frac{Z}{M^{\prime}} E_{j} \\
+\frac{i k_{j}}{M^{\prime} N} \int \frac{2 d^{3} p}{(2 \pi)^{3}} \zeta(\mathbf{p}) \delta f_{p}(\mathbf{k}, \omega)
\end{array}
$$

where $E_{j}$ is the electric field associated with vibrations, $N$ is the number of unit cells in $1 \mathrm{~cm}^{3}, M^{\prime}$ is the reduced mass of two atoms in the unit cell, and $Z$ is the effective ionic charge. The nonperturbed phonon frequency $\omega_{k}$ should be considered in the absence of the electric field and without any nonadiabatic corrections. In the longwave limit, we can roughly describe it as $\omega_{k}^{2}=\omega_{0}^{2} \pm s^{2} k^{2}$ with the magnitude of $s$ being of the order of the typical sound velocity in metals. The last term in Eq. (3) presents the driving force from the nonadiabatic electron system due to the deviation $\delta f_{p}(\mathbf{r}, t)$ from the localequilibrium distribution function $f_{0}[\varepsilon(\mathbf{p}, \mathbf{r}, t)-\mu]$.

Then, we have the Boltzmann equation

$$
\begin{array}{r}
-i(\omega-\mathbf{k} \cdot \mathbf{v}) \delta f_{p}(\mathbf{k}, \omega)+\gamma\left[\delta f_{p}(\mathbf{k}, \omega)-\left\langle\delta f_{p}(\mathbf{k}, \omega)\right\rangle\right] \\
=-[\omega \zeta(\mathbf{p}) \mathbf{k} \cdot \mathbf{u}(\mathbf{k}, \omega)+e \mathbf{v} \cdot \mathbf{E}] \frac{d f_{0}}{d \varepsilon}
\end{array}
$$

in the approximation of relaxation rate $\gamma$, which holds at low temperatures when the electron-impurity interaction dominates as well as at temperatures higher then the Debye temperature when the phonon-phonon collisions can be considered as elastic. The term in the angle brackets in Eq. (\#), which denote the average over the Fermi surface,

$$
\langle\ldots\rangle=\frac{1}{\nu_{0}} \int(\ldots) \frac{2 d S_{F}}{v(2 \pi)^{3}}
$$

arises from the out-term in the collision integral. Notice, that the condition $\langle\zeta(\mathbf{p})\rangle=0$ should be fulfilled, because the number of electrons in the local-equilibrium state $f_{0}[\varepsilon(\mathbf{p}, \mathbf{r}, t)-\mu]$ is conserved. With the help of the Maxwell equations, the electric field is expressed in the terms of polarization $\mathbf{P}$ as follows:

$$
\mathbf{E}=-4 \pi \mathbf{k}(\mathbf{k} \cdot \mathbf{P}) / k^{2},
$$

provided that phonons are excited in the optical region $k \gg \omega / c$ where the wavevector is determined by the incident light $k \sim \omega^{(i)} / c$ and the frequency $\omega$ is of the order of the optical phonon frequency $\omega_{0}$. It is seen that the electric field is longitudinal and only the longitudinal component of polarization $P_{z}$ ( $\mathbf{k}$ is taken along the $z$-axis) plays a role being related to the phonon displacement and the electric field by the equation

$$
P_{z}=N Z u_{z}+\alpha E+\frac{i e}{k} \int \frac{2 d^{3} p}{(2 \pi)^{3}} \delta f_{p}(\mathbf{k}, \omega),
$$

where the first term is caused by ionic motion, $\alpha$ is the polarizability of filled bands, and the last term is the carrier contribution defined by the variation of the electron density, $\rho^{(e)}=-i \mathbf{k} \cdot \mathbf{P}^{(e)}$.

Equations (3)-(6) give the complete system of our problem. The Boltzmann equation (4) has the solution in the form

$$
\delta f_{p}(\mathbf{k}, \omega)=-\chi_{p}(\mathbf{k}, \omega) \frac{d f_{0}}{d \varepsilon}
$$

where

$$
\begin{gathered}
\chi_{p}(\mathbf{k}, \omega)=i\left[e \mathbf{v} \cdot \mathbf{E}+\omega \zeta(\mathbf{p}) \mathbf{k} \cdot \mathbf{u}+\gamma\left\langle\chi_{p}(\mathbf{k}, \omega)\right\rangle\right] / \Delta,(7) \\
\left\langle\chi_{p}(\mathbf{k}, \omega)\right\rangle=i\langle[e \mathbf{v} \cdot \mathbf{E}+\omega \zeta(\mathbf{p}) \mathbf{k} \cdot \mathbf{u}] / \Delta\rangle /(1-i\langle\gamma / \Delta\rangle),
\end{gathered}
$$

and we set $\Delta=\omega-\mathbf{k} \cdot \mathbf{v}+i \gamma$. 
Using this solution, we obtain the polarization (6) and rewrite the electric field (5) in terms of the longitudinal displacement $u_{z}$ :

$$
\varepsilon_{e}(k, \omega) E=-4 \pi \beta_{f d} u_{z}
$$

where we introduce the field--displacement response function

$$
\beta_{f d}=N Z-e \omega \nu_{0} \frac{\langle\zeta(\mathbf{p}) / \Delta\rangle}{1-i\langle\gamma / \Delta\rangle}
$$

The electron contribution into the dielectric function has the known form:

$$
\varepsilon_{e}(k, \omega)-\varepsilon_{\infty}=-\frac{4 \pi e^{2} \nu_{0}\left\langle v_{z} / \Delta\right\rangle}{k(1-i\langle\gamma / \Delta\rangle)},
$$

where the high-frequency permittivity $\varepsilon_{\infty}=1+4 \pi \alpha$.

Now, we consider the equation of motion (3) using the solution of the Boltzmann equation (7). The term proportional to $u_{z}$ of the driving force can be included in the phonon frequency:

$$
\tilde{\omega}^{2}=\omega_{k}^{2}-i \omega \Gamma^{\mathrm{nat}}+\frac{\nu_{0} \omega k^{2}}{M^{\prime} N}\left(\left\langle\frac{\zeta^{2}(\mathbf{p})}{\Delta}\right\rangle+\frac{i \gamma\langle\zeta(\mathbf{p}) / \Delta\rangle^{2}}{1-i\langle\gamma / \Delta\rangle}\right),
$$

so that Eq. (3) reads

$$
\left(\tilde{\omega}^{2}-\omega^{2}\right) u_{z}=\tilde{Z} E / M^{\prime}
$$

where the renormalized ionic charge

$$
\tilde{Z}=Z-\frac{e \nu_{0} k}{N}\left(\left\langle\frac{\zeta(\mathbf{p}) v_{z}}{\Delta}\right\rangle+i \gamma \frac{\left\langle v_{z} / \Delta\right\rangle\langle\zeta(\mathbf{p}) / \Delta\rangle}{1-i\langle\gamma / \Delta\rangle}\right) .
$$

Using the condition $\langle\zeta(\mathbf{p})\rangle=0$ we obtain $\tilde{Z}=\beta_{f d} / N$.

Then, we can express the displacement $u_{z}$ from Eq. (12) in terms of $E$ and, substituting into Eq. (8), obtain the dielectric function of the electron-ion system:

$$
\varepsilon(k, \omega)=\varepsilon^{(e)}(k, \omega)+4 \pi N \tilde{Z}^{2} / M^{\prime}\left(\tilde{\omega}^{2}-\omega^{2}\right) .
$$

The frequency of the longitudinal mode, $\omega=\omega_{\mathrm{LO}}$, is defined by the condition $\varepsilon(k, \omega)=0$, i.e.,

$$
\omega^{2}=\tilde{\omega}^{2}+4 \pi N \tilde{Z}^{2} / M^{\prime} \varepsilon_{e}(k, \omega) .
$$

In the absence of free electrons, the density of states $\nu_{0}=0$ and Eq. (14) gives for the LO mode $\omega_{\mathrm{LO}}^{2}=\omega_{k}^{2}+$ $\omega_{p i}^{2}-i \omega_{\mathrm{L} 0} \Gamma^{\text {nat }}$, where $\omega_{p i}^{2}=4 \pi N Z^{2} / \varepsilon_{\infty}$ is the squared ion-plasma frequency of the order of $\omega_{0}^{2}$. For the TO mode, when the electric field $E=0$, we obtain $\omega_{\mathrm{TO}}^{2}=$ $\omega_{k}^{2}-i \omega_{k} \Gamma^{\text {nat }}$.

Free electrons in metals make the large contribution into the dielectric function [see, Eq. (10)]. Expanding in powers of $k$ we have in the zero-order

$$
\varepsilon^{(e)}(k, \omega)-\varepsilon_{\infty}=-\varepsilon_{\infty} \omega_{p e}^{2} / \omega(\omega+i \gamma),
$$

which corresponds simply to the Drude conductivity with the electron-plasma frequency

$$
\omega_{p e}^{2}=\frac{e^{2}}{3 \pi^{2} \varepsilon_{\infty}} \int v d S_{F} .
$$

For large $k v_{F}>|\omega+i \gamma|$, the electron contribution (10) describes the Debye screening:

$$
\varepsilon^{(e)}(k, \omega)-\varepsilon_{\infty}=\varepsilon_{\infty} \frac{k_{0}^{2}}{k^{2}}\left(1+i \pi \omega / 2 k v_{F}\right),
$$

where a term of the order of $\omega / k v$ is kept and the Debye parameter $k_{0}^{2}=4 \pi e^{2} \nu_{0} / \varepsilon_{\infty}$.

Therefore, we can solve Eq. (14) for $\omega_{L O} \ll \omega_{p e}$, using the iteration procedure. To a first approximation, we have

$$
\omega^{2}=\omega_{k}^{2}-i \omega_{k} \Gamma^{\text {nat }}+\frac{k^{2} \omega_{k} \nu_{0}}{M^{\prime} N}\left\langle\frac{\zeta^{2}(\mathbf{p})}{\Delta}\right\rangle+\frac{\omega_{p i}^{2} \varepsilon_{\infty}}{\varepsilon^{(e)}\left(k, \omega_{k}\right)} .
$$

In the case of small $k v_{F}<\left|\omega_{k}+i \gamma\right|$, expanding in $k$-powers, we obtain the solution

$$
\omega_{\mathrm{LO}}^{2}=\omega_{k}^{2}-i \omega_{k} \Gamma^{\mathrm{nat}}-\omega_{k}\left(\omega_{k}+i \gamma\right) \frac{\omega_{p i}^{2}}{\omega_{p e}^{2}}+\frac{\lambda \omega_{k} s^{2}}{\omega_{k}+i \gamma} k^{2},
$$

where the dimensionless coupling constant $\lambda=$ $\left\langle\zeta^{2}(\mathbf{p})\right\rangle \nu_{0} / \rho s^{2}$ contains the factor $a p_{F} m^{*} / m$ and the metal density $\rho$.

In the case of large $k$, expanding in $\left|\omega_{k}+i \gamma\right| / k v_{F}$, we obtain

$$
\omega_{L O}^{2}=\omega_{k}^{2}-i \omega_{k} \Gamma^{\mathrm{nat}}-i \frac{\pi \omega_{k} s^{2} k}{2 v_{F}}\left(\lambda+\frac{\omega_{p i}^{2}}{s^{2} k_{0}^{2}}\right)+\frac{\omega_{p i}^{2}}{k_{0}^{2}} k^{2},
$$

where the coupling constant $\lambda$ is defined, when the asymptotic value of the integral is calculated: $\nu_{0}\left\langle\zeta^{2}(\mathbf{p}) / \Delta\right\rangle / M^{\prime} N=-i \pi s^{2} \lambda / 2 k v_{F}$. Note, that the value of $\lambda$ vanishes in the isotropic case due to the con$\operatorname{dition}\langle\zeta(\mathbf{p})\rangle=0$.

Hence, the squared frequency of the longitudinal optical mode is essentially less (by the factor $\omega_{p i}^{2}$ ) than for insulators, due to screening of the electric field by free electrons. The additional phonon softening, width, and dispersion in Eq. (17) involve the adiabatic parameter $\left(\omega_{0} / \omega_{p e}\right)^{2} \sim m / M$, and they are small compared with $\Gamma^{\text {nat }} \sim \omega_{0} \sqrt{m / M}$. In the region, where Eq. (18) is valid, we see the additional $k$-dependent width (terms in the parentheses), which is comparable with $\Gamma^{\text {nat }}$. Here, the $\lambda$-term conditioned by the electron-phonon interaction is similar to the damping of the acoustic phonons [see the first formula in Eqs. (11)]. Now we omit the shift containing the small factor $\left(s / v_{F}\right)^{2}$. The second term in the parentheses as well as the last term in Eq. (18), giving the $k$-dispersion, are induced by screening and $\lambda$-independent. Since $\omega_{p i}^{2} / k_{0}^{2} \simeq s^{2}$, this dispersion has the typical value for the phonon branches. 
Let us rewrite in our notations the respective results of Ref. [8], Eqs. (9) and (7), retaining only main terms:

$$
\begin{gathered}
\omega_{\mathrm{LO}}^{2}=\omega_{k}^{2}-\frac{\omega_{p i}^{2}\left(\omega_{p i}^{2}+\omega_{k}^{2}\right)}{\omega_{p e}^{2}(1-2 \lambda \ln 2)}, \quad k v_{F}<\omega_{k}, \\
\omega_{\mathrm{LO}}^{2}=\omega_{k}^{2}+\frac{\omega_{p i}^{2}}{3 \omega_{p e}^{2}}\left(k v_{F}\right)^{2}, \quad k v_{F}>\omega_{k} .
\end{gathered}
$$

Comparing with Eqs. (17)-(18) we see that the $k$ dispersion coincides practically. Next, we agree that the contribution $\omega_{p i}^{2}$ vanishes from the frequency of the LO mode. The softening and damping due to both the electron-phonon scattering $(\gamma)$ and the phonon decayprocesses $\left(\Gamma^{\text {nat }}\right)$ were ignored in Ref. [8]. Concerning the electron-phonon interaction $\lambda$, the reason of disagreement was discussed in the beginning of the paper: this is shortcomings of diagram technique based on the Fröhlich model. But the most essential difference is the $k$-dependent width in Eq. (18), which is missed in Eq. (20).

In conclusion, let us make several remarks. The case of the large $k$-values (18) is most interesting because the electron-phonon and electrodynamic contributions into the phonon width (first and second terms in the parentheses, respectively) can compete. The result depends on the Debye screening and the wavevector $k$. In Raman experiments, the parameter $k v_{F} /\left|\omega_{0}+i \gamma\right| \sim \omega^{(i)} v_{F} / c \omega_{0} \simeq 0.3$ if $\omega^{(i)} \simeq 10^{4} \mathrm{~K}$ and $\omega_{0} \simeq 10^{2} \mathrm{~K}$, and for metals $v_{F} \simeq 10^{8}$ $\mathrm{cm} / \mathrm{s}$. Therefore, the high incident-light frequency or neutron experiments are desirable. It is more simply to observe the electrodynamic effect in semiconductors, where $k_{0}$ is smaller. We have an example of such experiments in works [11, where the metal-insulator transition was observed in the GaN crystal under pressure.
For the conducting phase, the longitudinal mode softens and obtains the additional damping in comparison with the insulator state. Then, using Eq. (17), we calculate 112 the collision rate $\gamma$ which is consistent with the value obtained from the conductivity.

The author thanks E. G. Mishchenko for fruitful discussions. The work was partially supported by the RFBR (project 01-02-16211).

[1] A.B. Migdal, Sov. Phys. JETP 7, 996 (1958).

[2] E.G. Brovman and Yu. Kagan, Sov. Phys. JETP 25, 365 (1967).

[3] B.T. Geilikman, J. Low Temp. Phys. 4, 189, (1971).

[4] M. Born and Kung Huang, Dynamical Theory of Crystal Lattices (Oxford University Press, New York, 1954).

[5] S. Engelsberg and J.R. Schrieffer, Phys. Rev. 131, 993 (1963).

[6] I.P. Ipatova and A.V. Subashiev, Sov. Phys. JETP 39, 349 (1974).

[7] A.S. Alexandrov and J.R. Schrieffer, Phys. Rev. B 56, 13731 (1997).

[8] M. Reizer, Phys. Rev. B 61, 40 (2000).

[9] V.M. Kontorovich, Sov. Phys. Uspekhi 27 (2), 134 (1984).

[10] L.A. Falkovsky and E.G. Mishchenko, Phys. Rev. B 51, 7239 (1995).

[11] P. Perlin, W. Knap, J. Camassel, A. Polian, J.C. Chervin, T. Suski, I. Grzegory, and S. Porowski, Phys. Stat. Sol. (b) 198, 223 (1996); P. Perlin, C. Jauberthie-Cariilon, J.P. Itie, A.San Miguel, I. Grzegory, and A. Polian, Phys. Rev. B 45, 83 (1992).

[12] L.A. Falkovsky, W. Knap, J.C. Chervin, and P. Wisniewski, Phys. Rev. B. 57, 11349 (1998). 